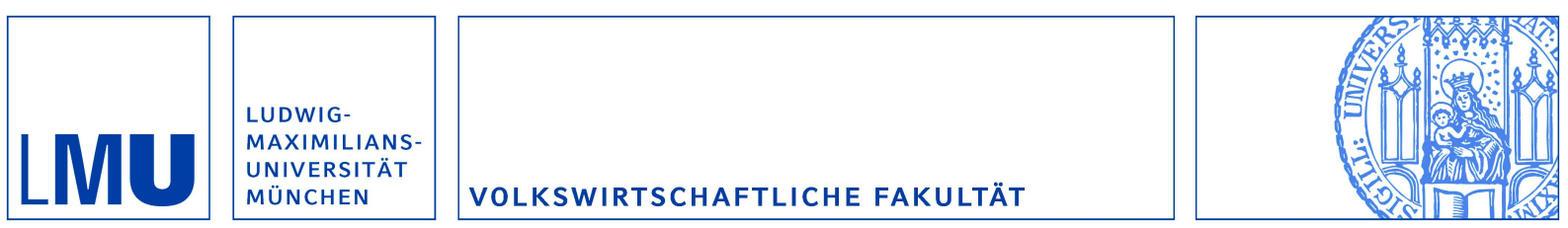

Ludwig, Sandra; Wichardt, Philipp C. und Wickhorst, Hanke:

Overconfidence Can Improve an Agent's Relative and Absolute Performance in Contests

Munich Discussion Paper No. 2010-35

Department of Economics

University of Munich

Volkswirtschaftliche Fakultät

Ludwig-Maximilians-Universität München

Online at https://doi.org/10.5282/ubm/epub. 11885 


\title{
Overconfidence Can Improve an Agent's Relative and Absolute Performance in Contests*
}

\author{
Sandra Ludwig ${ }^{\dagger}$ \\ Dept. of Economics, LMU Munich \\ Philipp C. Wichardt \\ Dept. of Economics, University of Bonn \\ Hanke Wickhorst ${ }^{\S}$ \\ Münster School of Business and Economics
}

This Version: July 5, 2010

\begin{abstract}
This paper suggests a potential rationale for the recent empirical finding that overconfident agents tend to self-select into more competitive environments (e.g. Dohmen and Falk, forthcoming). In particular, it shows that moderate overconfidence in a contest can improve the agent's performance relative to an unbiased opponent and can even lead to an advantage for the overconfident agent in absolute terms.
\end{abstract}

Keywords: Overconfidence, Contests.

JEL classification: D21, D44, D82.

*Acknowledgments: We would like to thank Alexander Dilger, several participants of the ESA European Meeting 2009 and an anonymous reviewer for helpful comments and suggestions. Financial support from the German Research Foundation (DFG), SFB/TR 15 at the University of Munich, is gratefully acknowledged.

${ }_{\dagger}$ e-mail: sandra.ludwig@lrz.uni-muenchen.de

‡e-mail: philipp.wichardt@uni-bonn.de.

$\S$ Postal address for correspondence: Institute for Economic Education, University of Münster, Scharnhorststr. 100, D-48151 Münster, Germany; tel.: +49 (0)251 83 25339, fax: +49 (0)251 83 28429, e-mail: hanke.wickhorst@uni-muenster.de. 


\section{Introduction}

Recent empirical evidence shows that overconfident agents tend to self-select into more competitive environments than unbiased agents (e.g. Dohmen and Falk, forthcoming; Bartling et al., 2009). At first sight, this may seem puzzling as overconfidence in contests is commonly found to reduce individual welfare due to suboptimally high effort choices, which - if at all - are beneficial for the principal (cf. Santos-Pinto, 2010; Ando, 2004). ${ }^{1}$

However, an effect that seems to have gone unnoticed in the literature on overconfidence in contests is that the high effort of the overconfident agent may also lead to a comparative payoff-advantage of the biased agent due to an increased probability of success. In fact, although both Ando (2004) and Santos-Pinto (2010) touch on individual welfare effects of overconfidence in contests, neither of them considers relative payoff effects in their analysis (and we are not aware of any other paper that does so). Yet, as shown below, the induced increase in the probability of success of an overconfident agent may not only reverse the relative performance of the agents. It may even overcompensate the biased agent for his additional effort and, thereby, increase his payoff above the rational benchmark (if the bias is sufficiently small). Thus, overconfident agents may actually be correct in believing that they have a comparative advantage in more competitive environments such as economic contests.

In the sequel, we demonstrate these positive effects of overconfidence in a simple model of imperfectly discriminating contests in the tradition of Tullock (1980).

\section{$2 \quad$ Model and Results}

The Model. Consider a standard two agent Tullock contest with linear effort costs where agents compete for a winner-price $w_{H}$ (the loser gets $w_{L}$, with $\Delta w=w_{H}-$ $\left.w_{L}\right) .^{2}$ In order to simplify the exposition, we restrict attention to a contest success function with a discriminatory power of 1 . While not affecting the general thrust of the argument, the assumption, for example, guarantees the existence of equilibrium. Moreover, assume that one agent (agent 1) is overconfident while the other (agent 2) is rational. In particular, to capture agent 1's overly optimistic view on his abilities,

\footnotetext{
${ }^{1}$ See, e.g., Yates (1990) for some background on the discussion of overconfidence in psychology.

${ }^{2}$ Assuming effort costs to be linear essentially simplifies the subsequent exposition but is not crucial for the qualitative results to be derived.
} 
assume that he has a biased perception of his effort cost, i.e. $\tilde{c}_{1}=c_{1}-b$ where $c_{1}$ is agent 1's true cost of effort and $0<b<c_{1}$. The rational agent 2 , by contrast, has a correct view about his effort cost, i.e. $\tilde{c}_{2}=c_{2}>0$. The resulting maximisation problem of agent $i$, then, is given by:

$$
\max _{e_{i}} \frac{e_{i}}{e_{i}+e_{-i}} \Delta w+w_{L}-\tilde{c}_{i} e_{i}
$$

which gives rise to concave reaction functions with positive (negative) first derivatives for small (large) values of $e_{-i}$. For the case of $e_{1}=e_{2}=0$, assume that each agent wins the contest with probability 0.5. Again, the assumption is not restrictive as in equilibrium both agents will exert strictly positive effort.

Finally, assume that both agents are informed about their own perceived effort $\operatorname{cost} \tilde{c}_{i}$ (but not about a potential own bias) and the perceived effort cost of their opponent $\tilde{c}_{-i}$; for the rational agent this is equivalent to assuming that he knows his true effort cost as $\tilde{c}_{1}=c_{1}$. In effect, the assumption ensures that both agents best respond to their opponent's action so that attention is restricted entirely to the effects of overconfidence while informational issues are set aside. ${ }^{3}$ A standard argument, then, shows that the corresponding Nash equilibrium effort levels are:

$$
e_{1}^{*}=\frac{\Delta w c_{2}}{\left(\tilde{c}_{1}+c_{2}\right)^{2}}=\frac{\Delta w c_{2}}{\left(c_{1}+c_{2}-b\right)^{2}}
$$

and

$$
e_{2}^{*}=\frac{\Delta w \tilde{c}_{1}}{\left(\tilde{c}_{1}+c_{2}\right)^{2}}=\frac{\Delta w\left(c_{1}-b\right)}{\left(c_{1}+c_{2}-b\right)^{2}} .
$$

Thus, if both agents are rational, i.e. $b=0$, equilibrium effort levels are:

$$
e_{1}^{B M}=\frac{\Delta w c_{2}}{\left(c_{1}+c_{2}\right)^{2}} \quad \text { and } \quad e_{2}^{B M}=\frac{\Delta w c_{1}}{\left(c_{1}+c_{2}\right)^{2}}
$$

which we will consider as the benchmark for our analysis.

Aggregate Effects. To begin with, note that irrespective of agent 2's effort cost, agent 1 's effort increases in his bias because $\frac{\partial e_{1}^{*}}{\partial b}=\frac{2 \Delta w c_{2}}{\left(c_{1}+c_{2}-b\right)^{3}}>0$ (as $\left.b<c_{1}\right)$. The effort of

\footnotetext{
${ }^{3}$ From an applied point of view, assuming agents to best respond to each other's actions seems reasonable as an approximation, for example, in settings where some ex ante learning about the opponent's reaction function is possible, e.g. because information about past behaviour is readily available. Such learning need not affect the biased self-perception of overconfident agents, though, as for example Pulford and Colman (1997) show that for such agents feedback about eventual outcomes essentially leaves their biases unaffected.
} 
the rational agent 2 , by contrast, decreases in agent 1 's overconfidence if agent 1 's perceived effort cost is smaller than agent 2's true cost, i.e. if $\tilde{c}_{1}<c_{2}$ it holds that $\frac{\partial e_{2}^{*}}{\partial b}=\frac{\Delta w\left(c_{1}-c_{2}-b\right)}{\left(c_{1}+c_{2}-b\right)^{3}}<0$ (as $\left.b<c_{1}\right)$; it increases otherwise. Similar to previous results (e.g. Santos-Pinto, 2010), combining these two effects yields that overconfidence is beneficial for the principal (who wants to maximise aggregate efforts) as the sum of the efforts in the case with overconfidence (equations (2) and (3)) is greater than the sum of the efforts in the rational benchmark (equations (4)): $e_{1}^{*}+e_{2}^{*}>e_{1}^{B M}+e_{2}^{B M} .4$

Individual Effects. In order to assess the relative individual payoff effects, consider the agents' expected equilibrium payoffs. These are given by:

$$
U_{i}^{*}=\frac{e_{i}^{*}}{e_{i}^{*}+e_{-i}^{*}} \Delta w+w_{L}-c_{i} e_{i}^{*}
$$

where the true effort cost has to be taken into account. Accordingly, the payoff difference between the overconfident agent 1 and the rational agent 2 is:

$$
\Delta U^{*}:=U_{1}^{*}-U_{2}^{*}=\frac{e_{1}^{*}-e_{2}^{*}}{e_{1}^{*}+e_{2}^{*}} \Delta w-c_{1} e_{1}^{*}+c_{2} e_{2}^{*}
$$

Inserting equations (2) and (3) into (6) gives:

$$
\Delta U^{*}=\frac{\Delta w}{\left(\tilde{c_{1}}+c_{2}\right)^{2}}\left[c_{2}\left(c_{2}+\tilde{c_{1}}-c_{1}\right)-{\tilde{c_{1}}}^{2}\right]=\frac{\Delta w}{\left(c_{1}+c_{2}-b\right)^{2}}\left[c_{2}\left(c_{2}-b\right)-\left(c_{1}-b\right)^{2}\right] .
$$

Calculating the first derivative with respect to $b$ shows that becoming more overconfident increases agent 1's relative performance compared to agent 2 if the bias is moderate, $b<\frac{c_{1}+c_{2}}{3}:^{5}$

$$
\begin{aligned}
\frac{\partial \Delta U^{*}}{\partial b} & =\frac{\Delta w}{\left(c_{1}+c_{2}-b\right)^{3}}\left[\left(c_{1}+c_{2}-b\right)\left(-c_{2}+2\left(c_{1}-b\right)\right)+2\left(c_{2}\left(c_{2}-b\right)-\left(c_{1}-b\right)^{2}\right)\right] \\
& =\frac{\Delta w c_{2}}{\left(c_{1}+c_{2}-b\right)^{3}}\left(c_{1}+c_{2}-3 b\right) .
\end{aligned}
$$

Furthermore, it follows from (7) that

$$
\Delta U^{*} \geq 0, \text { if }\left\{\begin{array}{l}
\tilde{c_{1}}<c_{1} \leq c_{2}, \\
\tilde{c_{1}}<c_{2}<c_{1} \text { and } c_{2} \geq \frac{4}{5} c_{1} \text { and } b \in\left[b_{1}, b_{2}\right]
\end{array}\right.
$$

\footnotetext{
${ }^{4}$ Similarly, underconfidence (i.e. $b<0$ and agent 1 overestimates his effort cost) is detrimental for the principal.

${ }^{5}$ The derivative is also positive if $b>c_{1}+c_{2}$ but this can never hold as $b<c_{1}$ and $c_{i}>0$.
} 
where $b_{1}=\frac{2 c_{1}-c_{2}-\sqrt{c_{2}\left(5 c_{2}-4 c_{1}\right)}}{2}$ and $b_{2}=\frac{2 c_{1}-c_{2}+\sqrt{c_{2}\left(5 c_{2}-4 c_{1}\right)}}{2} \cdot{ }^{6}$ Hence, a necessary condition for $U_{2}^{*}>U_{1}^{*}$ to hold, i.e. for $\Delta U^{*}<0$, is that $c_{2}<c_{1}$. In particular, $U_{2}^{*}>U_{1}^{*}$ holds irrespective of the size of $b$ if agent 2 is sufficiently more skilled than agent 1, i.e. if $c_{2}<\frac{4}{5} c_{1}$, otherwise, i.e. if $\frac{4}{5} c_{1} \leq c_{2}<c_{1}, U_{2}^{*}>U_{1}^{*}$ holds only if $b \notin\left[b_{1}, b_{2}\right]$. Put differently, if agent 1 is at least as skilled as agent $2, \Delta U^{*}$ is positive irrespective of the level of overconfidence; see Figure 1 for an illustration.

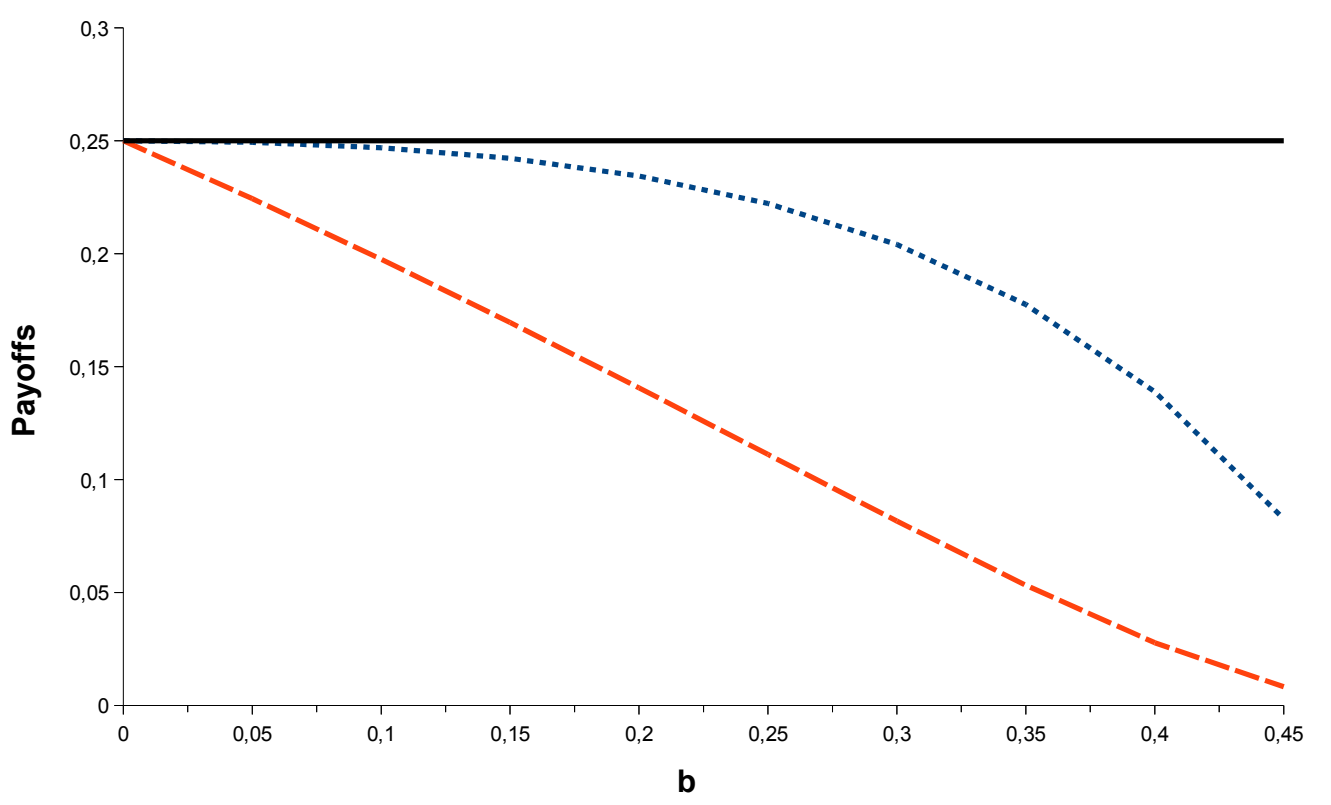

Figure 1: Expected equilibrium payoffs as a function of $b$ for parameters: $c_{1}=c_{2}=$ $0.5 ; \Delta w=1$ and $b \in[0,0.45]$. The continuous line shows an agent's expected payoff in equilibrium if both agents are rational. The other lines refer to the expected payoffs of the overconfident agent (dotted line) and the rational agent (dashed line) in the contest with one rational and one overconfident agent.

Moreover, the comparative advantage of agent 1 can persist even if agent 2 is the more skilled agent (cf. equation (9)). Accordingly, overconfidence can lead to a situation where the biased agent 1 has a greater chance of winning the contest despite being the less able one. In this case, the induced increase in agent 1's effort leads to an increase in the winning probability that outweighs agent 1's higher cost;

\footnotetext{
${ }^{6}$ When $\tilde{c_{1}}<c_{2}<c_{1}$, we solve $c_{2}\left(c_{2}-b\right)-\left(c_{1}-b\right)^{2} \geq 0$ for $b$ (cf. equation (7)). The inequality is quadratic in $b$, and $b_{1}$ and $b_{2}$ are the two existing zeros for $c_{2} \geq \frac{4}{5} c_{1}$. Both $b_{1}$ and $b_{2}$ are in the feasible range, i.e. $0<b_{1}<b_{2}<c_{1}$. Moreover, the inequality holds (implying that $\Delta U^{*}$ is positive) if $b \in\left[b_{1}, b_{2}\right]$; otherwise the inequality is violated.
} 
thus agent 1 is better off than his opponent, although he exerts the higher effort and has the higher effort cost per unit of effort. ${ }^{7}$

Finally, a comparison of agent 1's payoff for the case of $b>0$ with that of the benchmark scenario, $b=0$, shows that being overconfident may indeed even improve agent 1's absolute payoff as

$$
U_{1}^{*}-U_{1}^{B M}>0 \quad \text { if } \quad \frac{c_{2}^{2}-c_{1}^{2}}{c_{2}}>b,
$$

i.e. if agent 1 is the more skilled agent $\left(c_{2}>c_{1}\right)$ and the bias is sufficiently small. To see that (10) holds, note that:

$$
\begin{aligned}
U_{1}^{*}-U_{1}^{B M} & =\frac{\Delta w c_{2}\left(c_{1}+c_{2}-b\right)-\Delta w c_{1} c_{2}}{\left(c_{1}+c_{2}-b\right)^{2}}+\frac{\Delta w c_{1} c_{2}-\Delta w c_{2}\left(c_{1}+c_{2}\right)}{\left(c_{1}+c_{2}\right)^{2}} \\
& =\frac{\left(\Delta w c_{2}^{2}-\Delta w c_{2} b\right)\left(c_{1}+c_{2}\right)^{2}-\Delta w c_{2}^{2}\left(c_{1}+c_{2}-b\right)^{2}}{\left(c_{1}+c_{2}\right)^{2}\left(c_{1}+c_{2}-b\right)^{2}} \\
& =\frac{b \Delta w c_{2}}{\left(c_{1}+c_{2}\right)^{2}\left(c_{1}+c_{2}-b\right)^{2}}\left[c_{2}^{2}-c_{1}^{2}-c_{2} b\right]
\end{aligned}
$$

which is positive if $\frac{c_{2}^{2}-c_{1}^{2}}{c_{2}}>b$.

By contrast, compared to the benchmark situation with $b=0$, the rational agent 2 is always worse off when paired with an overconfident agent $1(b>0)$, i.e.

$$
U_{2}^{*}-U_{2}^{B M}<0
$$

because

$$
\begin{aligned}
U_{2}^{*}-U_{2}^{B M}= & \frac{\left(\Delta w c_{1}-\Delta b\right)\left(c_{1}+c_{2}-b\right)-\Delta w c_{2}\left(c_{1}-b\right)}{\left(c_{1}+c_{2}-b\right)^{2}} \\
& +\frac{\Delta w c_{1} c_{2}-\Delta w c_{1}\left(c_{1}+c_{2}\right)}{\left(c_{1}+c_{2}\right)^{2}} \\
= & \frac{\Delta w c_{2} b}{\left(c_{1}+c_{2}\right)^{2}\left(c_{1}+c_{2}-b\right)^{2}}\left[2 c_{1} b+c_{2} b-2 c_{1} c_{2}-2 c_{1}^{2}\right]
\end{aligned}
$$

is always smaller than zero as $b<c_{1}$.

Proposition 1 below summarises the main points of the above analysis.

\footnotetext{
${ }^{7}$ If agent 1 is instead underconfident, i.e. $b<0$, all effects are reversed: Becoming less underconfident increases agent 1 's effort and his performance relative to agent 2 , and $\Delta U^{*}>0$ is only possible if agent 1 is the more able agent.
} 
Proposition 1 For the above described Tullock-contest between an overconfident agent 1 with a strictly positive bias $(b>0)$ and a rational agent 2 , it holds that:

1. The principal is strictly better off than in the case without overconfidence $(b=$ 0) as $e_{1}^{*}+e_{2}^{*}>e_{1}^{B M}+e_{2}^{B M}$.

2. Agent 1 overexerts effort with respect to his ability, i.e. $\frac{\partial e_{1}^{*}}{\partial b}>0$.

3. Agent 2 reduces his effort compared to the rational benchmark $(b=0)$, i.e. $\frac{\partial e_{2}^{*}}{\partial b}<0$, if $c_{1}-c_{2}<b$.

4. Agent 2 is always worse off if agent 1 is overconfident than if he is not, i.e. $U_{2}^{*}<U_{2}^{B M}$.

5. For small biases $\left(b<\frac{c_{1}+c_{2}}{3}\right)$, agent 1's relative performance as measured by the difference in equilibrium payoffs $\Delta U^{*}=U_{1}^{*}-U_{2}^{*}$ is increasing in $b$.

6. Agent 1's equilibrium payoff is larger than that of agent 2, i.e. $\Delta U^{*}>0$, if $c_{1} \leq c_{2}$, or if $c_{1}>c_{2} \geq \frac{4}{5} c_{1}$ and $b \in\left[b_{1}, b_{2}\right]$.

7. Agent 1 has an absolute payoff advantage from being overconfident if his true cost of effort is smaller than that of agent 2 and if his bias is small, i.e. $U_{1}^{*}>U_{1}^{B M}$ for $c_{1}<c_{2}$ and $0<b<\frac{c_{2}^{2}-c_{1}^{2}}{c_{2}}$.

Extension to two overconfident agents. As a last step of the analysis, we briefly consider the case of two overconfident agents with different biases but identical true effort cost $c$. For the sake of argument, we assume that agent 1's bias is larger than the bias of agent 2, i.e. $0<b_{2}<b_{1}<c .8$ In this case, assuming as before that both agents know their own perceived cost $\tilde{c}_{i}$ and the perceived cost of their opponent $\tilde{c}_{-i}$ and solving the corresponding maximisation problem described in equation (1), we obtain the following equilibrium effort levels:

$$
\hat{e}_{1}=\frac{\Delta w\left(c-b_{2}\right)}{\left(2 c-b_{1}-b_{2}\right)^{2}} \quad \text { and } \quad \hat{e}_{2}=\frac{\Delta w\left(c-b_{1}\right)}{\left(2 c-b_{1}-b_{2}\right)^{2}}
$$

Moreover, inserting equilibrium efforts into the agents' utility functions (cf. equation (5)), the expression for $\Delta U$ becomes:

$$
\Delta \hat{U}:=\hat{U}_{1}-\hat{U}_{2}=\underbrace{\frac{\Delta w}{\left(2 c-b_{1}-b_{2}\right)^{2}}}_{>0}\left[b_{2}^{2}-b_{1}^{2}+c\left(b_{1}-b_{2}\right)\right] .
$$

\footnotetext{
${ }^{8}$ If $b_{1}=b_{2}$, both agents trivially have the same expected payoffs as the maximisation problem is symmetric.
} 
And, as $\Delta w>0$, the condition for the more biased agent 1 to be better off, $\Delta \hat{U}>0$, can be simplified to

$$
\left(b_{1}-b_{2}\right)\left(c-\left(b_{1}+b_{2}\right)\right)>0 \text {. }
$$

Thus, the more biased agent 1 is better off (worse off) than agent 2 whenever the sum of the biases is smaller (larger) than $c$.

Moreover, regarding comparative effects, if $c=b_{1}+b_{2}$ (so that $\Delta \hat{U}=0$ ) but $b_{2}<b_{1},{ }^{9}$ the more biased agent 1 , taking $b_{2}$ as given, would gain a comparative advantage from having a lower bias. In particular, if $b_{1}$ was lowered while keeping $b_{2}$ fixed, the situation would change to one with $c>b_{1}+b_{2}$ so that $\Delta \hat{U}$ would become negative provided that $b_{1}$ remains larger than $b_{2}$. Similarly, taking $b_{1}$ as given, the less biased agent 2 would gain a comparative advantage from an increase in his bias so that $c<b_{1}+b_{2}$ while keeping $b_{2}<b_{1}$. Accordingly, if also biases were a matter of choice, the only stable situation would be the one with $c=b_{1}+b_{2}$ and $b_{1}=b_{2}$ : if $b_{i}$ was increased (decreased) in such a case, the situation would change to one with $b_{i}>b_{-i}$ and $c<b_{i}+b_{-i}\left(b_{i}<b_{-i}\right.$ and $\left.c>b_{i}+b_{-i}\right)$ so that agent $i$ would be worse off compared to his opponent. Put differently, while both agents always have the same expected payoffs if $b_{1}=b_{2}$, it is only when $b_{1}=b_{2}$ and also $b_{1}+b_{2}=c$ that no agent would generate a comparative advantage from having a different level of overconfidence.

Proposition 2 For the above described Tullock-contest between two overconfident agents with identical true effort cost $c$ and biases $0<b_{i}<c, i=1,2$, it holds that:

1. The agents expected payoffs are identical whenever $b_{1}=b_{2}$ or $b_{1}+b_{2}=c$.

2. If $b_{1}+b_{2}<c$ and $b_{1} \neq b_{2}$, the expected payoff is larger for the agent with the larger bias.

3. If $b_{1}+b_{2}>c$ and $b_{1} \neq b_{2}$, the expected payoff is larger for the agent with the smaller bias.

4. If $b_{1}+b_{2}=c$ and $b_{1}=b_{2}$ (and only then), no agent would gain a comparative advantage over his opponent from a change in his bias.

Finally, it deserves a mention that the continuity of the agents' reaction and utility functions ensures that the main results of the analysis - (a) that overconfidence may outweigh a higher cost of effort (cf. Proposition 1.6) and (b) that the

\footnotetext{
${ }^{9}$ See Footnote 8.
} 
relative advantage of being more (or less) overconfident in the case with two biased agents and equal true effort cost depends on the sum of the biases (cf. Proposition 2) - also transfer to the more general case of two overconfident agents with different effort cost (at least for some range of parameters).

\section{References}

Ando, M. (2004): Overconfidence in Economic Contests, Working Paper, Nihon University.

Bartling, B., E. Fehr, M.A. Marchal, and D. Schunk (2009), "Egalitarianism and Competitiveness," American Economic Review (Papers and Proceedings), 99, 93-98.

Dohmen, T., and A. Falk (forthcoming), "Performance Pay and Multi-dimensional Sorting: Productivity, Preferences and Gender," American Economic Review.

Santos-Pinto, L. (2010): Positive Self-Image in Tournaments, International Economic Review, 51, 475-496.

Pulford, B. and A. Colman (1997). "Overconfidence: Feedback and Item Difficulty Effects," Personality and Individual Differences, 23, 125-133.

Tullock, G. (1980): "Efficient Rent-Seeking," pp. 97-112 in: Buchanan, J.M. Tollison, R.D. und Tullock, G. (Ed.), Toward a Theory of the Rent-Seeking Society, College Station: Texas A\&M University Press.

Yates, J.F. (1990): Judgement and Decision Making, Englewood Cliffs, NJ: Prentice Hall. 